\title{
DVCS with a Longitudinally Polarized Target with CLAS12
}

\author{
Hovanes Egiyan
}

Angela Biselli

Fairfield University, abiselli@fairfield.edu

CLAS Collaboration

Follow this and additional works at: https://digitalcommons.fairfield.edu/physics-facultypubs

Copyright 2009 AIP Publishing

This article may be downloaded for personal use only. Any other use requires prior permission of the author and AIP Publishing. The following article appeared in Egiyan, H., Biselli, A., \& CLAS Collaboration. (2009, August). DVCS with a longitudinally polarized target with CLAS12. In AIP Conference Proceedings (Vol. 1149, No. 1, pp. 607-610). AIP. and may be found at https://doi.org/10.1063/1.3215720.

The final publisher PDF has been archived here with permission from the copyright holder.

\section{Repository Citation}

Egiyan, Hovanes; Biselli, Angela; and CLAS Collaboration, "DVCS with a Longitudinally Polarized Target with CLAS12" (2009). Physics Faculty Publications. 136.

https://digitalcommons.fairfield.edu/physics-facultypubs/136

\section{Published Citation}

Egiyan, H., Biselli, A., \& CLAS Collaboration. (2009, August). DVCS with a longitudinally polarized target with CLAS12. In AIP Conference Proceedings (Vol. 1149, No. 1, pp. 607-610). AIP. doi:10.1063/1.3215720.

This item has been accepted for inclusion in DigitalCommons@Fairfield by an authorized administrator of DigitalCommons@Fairfield. It is brought to you by DigitalCommons@Fairfield with permission from the rightsholder(s) and is protected by copyright and/or related rights. You are free to use this item in any way that is permitted by the copyright and related rights legislation that applies to your use. For other uses, you need to obtain permission from the rights-holder(s) directly, unless additional rights are indicated by a Creative Commons license in the record and/or on the work itself. For more information, please contact digitalcommons@fairfield.edu. 


\section{DVCS with a Longitudinally Polarized Target with CLAS12}

Hovanes Egiyan, Angela Biselli, and CLAS Collaboration

Citation: AIP Conference Proceedings 1149, 607 (2009); doi: 10.1063/1.3215720

View online: https://doi.org/10.1063/1.3215720

View Table of Contents: http://aip.scitation.org/toc/apc/1149/1

Published by the American Institute of Physics 


\title{
DVCS with a Longitudinally Polarized Target with CLAS12
}

\author{
Hovanes Egiyan*, Angela Biselli ${ }^{\dagger}$ and the CLAS Collaboration** \\ ${ }^{*}$ University of New Hampshire, New Hampshire, 03824, USA \\ ${ }^{\dagger}$ Fairfield University, Connecticut, 06824, USA \\ ${ }^{* *}$ Newport News, Virginia, 23606, USA
}

\begin{abstract}
We describe a planned experiment to measure the longitudinal target-spin asymmetry in the DVCS process using CLAS12 detector. While the beam-spin asymmetry is more sensitive to $H$ GPD, measurements of the target-spin asymmetry allow us to access the $\widetilde{H}$ GPD. These data will extend the $Q^{2}$ range accessable by the existing CLAS data and the scheduled 6 GeV CLAS DVCS experiments to $Q^{2}=8 \mathrm{GeV}^{2}$.
\end{abstract}

Keywords: DVCS, polarized, target, GPD, Compton, formfactor, asymmetry

PACS: $13.60 . \mathrm{Fz}, 14.20 . \mathrm{Dh}, 13.88 .+\mathrm{e}, 13.40 . \mathrm{Gp}$

\section{INTRODUCTION}

The recently introduced Generalized Parton Distributions (GPD) formalism [1, 2, 3, 4] provides us with a unifying framework to describe the nucleon structure. These functions contain the usual form factors and parton distributions, but in addition they include correlations between states of different longitudinal momentum and transverse momentum dependence. GPDs can therefore give three-dimensional pictures of the nucleon, providing information such as the transverse spatial distribution as a function of the longitudinal momentum fraction of the quarks.

Deeply Virtual Compton Scattering (DVCS) is the simplest and, therefore, the cleanest process which can be described in terms of GPDs. It was shown $[1,2,5,3,4]$ that the DVCS reaction $\gamma^{*} p \rightarrow \gamma p$, in the Bjorken limit, can be factorized into a hard scattering kernel and a non-perturbative part (see Fig.1 left), described at twist-2 level by four GPDs $E, H, \widetilde{E}$ and $\widetilde{H}$. This factorization is valid when the virtuality of the incoming photon is large, but the momentum transfer to the nucleon is small $\left(-t<<Q^{2}\right)$. The GPDs are functions of three variables $x, \xi$ and $t$, where $x$ characterizes the average light-cone momentum fraction of the struck quark in the loop, $\xi$ is the longitudinal momentum fraction of the transfer to the proton $\Delta=p-p^{\prime}$, and $t=\Delta^{2}$ is the standard transfer between the virtual and real photons. Recent results of DVCS Beam Spin Asymmetries (BSA) from Jefferson Lab and HERMES $[6,7,8,9,10]$ suggested that the GPD framework can be applied to DVCS experiments at the electron beam energies as low as $6 \mathrm{GeV}$.

In the forward limit $(\xi=t=0)$, the GPDs $H$ and $\widetilde{H}$ reduce to the usual parton distributions $q(x)$ and $\Delta q(x)$ :

$$
H^{q}(x, \xi=0, t=0)=q(x),
$$




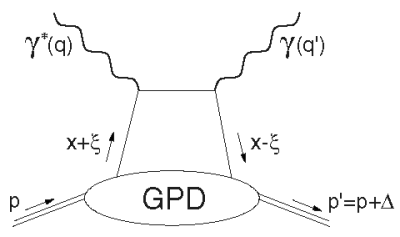

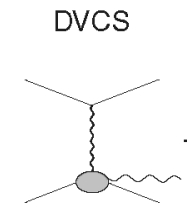

(a)

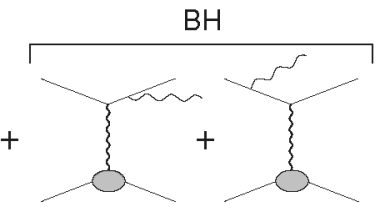

(b)

(c)

FIGURE 1. Handbag diagram to the DVCS process (left); diagrams contributing to the photon electroproduction process(right).

$$
\widetilde{H}^{q}(x, \xi=0, t=0)=\Delta q(x)
$$

where $q$ stands for the quark flavor. The first moments of the GPDs and the elastic form factors are related by the following relations:

$$
\begin{aligned}
& \int_{-1}^{+1} d x H^{q}(x, \xi, t)=F_{1}^{q}(t), \quad \int_{-1}^{+1} d x E^{q}(x, \xi, t)=F_{2}^{q}(t), \\
& \int_{-1}^{+1} d x \widetilde{H}^{q}(x, \xi, t)=g_{A}^{q}(t), \quad \int_{-1}^{+1} d x \widetilde{E}^{q}(x, \xi, t)=h_{A}^{q}(t),
\end{aligned}
$$

where $F_{1}^{q}$ and $F_{2}^{q}$ are the Dirac and Pauli form factors, $g_{A}^{q}$ is the axial form factor and $h_{A}^{q}$ is the induced pseudoscalar form factor. The second moment of the GPDs and the total angular momentum carried by quarks are related via Ji's sum rule:

$$
\sum_{q} \frac{1}{2} \int_{-1}^{+1} d x x\left[H^{q}(x, \xi, t=0)+E^{q}(x, \xi, t=0)\right]=J_{q} .
$$

Since $\Delta \Sigma$ is constrained in DIS experiments, if one makes enough measurements to extract the second moments of the GPDs, the sum rule will determine the quark orbital momentum contribution to the nucleon spin.

The $e p \rightarrow e p \gamma$ process can either occur by radiation along one of the electron lines (Bethe-Heitler or BH) or by emission of a real photon by the nucleon (DVCS) as shown in right panel of Fig. 1. The total amplitude $\mathscr{T}$ is the coherent sum of the BH and DVCS amplitudes:

$$
\begin{aligned}
|\mathscr{T}|^{2} & =\left|\mathscr{T}_{B H}\right|^{2}+\left|\mathscr{T}_{D V C S}\right|^{2}+\mathscr{I} \\
\mathscr{I} & =\mathscr{T}_{D V C S}^{*} \mathscr{T}_{B H}+\mathscr{T}_{D V C S} \mathscr{T}_{B H}^{*}
\end{aligned}
$$

where $\mathscr{T}_{D V C S}$ and $\mathscr{T}_{B H}$ are the amplitudes for the DVCS and Bethe-Heitler processes, and $\mathscr{I}$ denotes the interference between these amplitudes. The $\left|\mathscr{T}_{D V C S}\right|^{2}$ term is predicted to be smaller than other contributions, and therefore we do not keep it in this discussion. On the other hand, it may be possible to make empirical estimates of the relative magnitude of the $\left|\mathscr{T}_{D V C S}\right|^{2}$ term using future accurate data. Neglecting the $\left|\mathscr{T}_{D V C S}\right|^{2}$ term, the longitudinal target-spin asymmetry can be expressed in terms of Compton 
formfactors (CFFs) as:

$$
\begin{aligned}
A_{L U} & =\frac{d \sigma^{\rightarrow}-d \sigma^{\leftarrow}}{\sigma^{\rightarrow}+d \sigma^{\leftarrow}}=\frac{2 \cdot \mathscr{T}_{B H} \cdot \operatorname{Im}\left(\mathscr{T}_{D V C S}\right)}{\left|T_{B H}\right|^{2}+2 \cdot \mathscr{T}_{B H} \cdot \operatorname{Re}\left(\mathscr{T}_{D V C S}\right)}= \\
& =\left\{F_{1} \widetilde{\mathscr{H}}+\frac{x_{B}}{2-x_{B}}\left(F_{1}+F_{2}\right)\left(\mathscr{H}+\frac{x_{B}}{2} \mathscr{E}\right)-\frac{x_{B}}{2-x_{B}}\left(\frac{x_{B}}{2} F_{1}+\frac{t}{4 M^{2}} F_{2}\right) \widetilde{\mathscr{E}}\right\}
\end{aligned}
$$

We can see from Eq. 8 that the main contributions to $A_{L U}$ come from $\widetilde{\mathscr{H}}$ and $\mathscr{H}$, whereas the $\widetilde{\mathscr{E}}$ contribution is suppressed. Clearly we need at least another measured observable, for instance the beam-spin asymmetry, to be able to separate the $\widetilde{\mathscr{H}}$ and $\mathscr{H}$ terms. The extraction of GPDs will be done in the same manner as Parton Distribution Functions are extracted from structure function measurements, by means of a global fit of data using GPD parametrization.

\section{EXPERIMENTAL DATA}

The proposed experiments will use the standard CLAS12 setup. Six symmetrically arranged superconducting coils will provide a toroidal magnetic field for the spectrometer. Each of the six sectors of CLAS12 will be instrumented with identical detector packages, consisting of drift chambers for tracking, Cherenkov counters and time-of-flight systems for particle identification, electromagnetic shower and pre-shower calorimeters for triggering and neutral particle detection. A central detector system, consisting of a silicon vertex detector and a time-of-flight system will provide us with a detection and identification capabilities for slower backward-going particles. A special lead-glass calorimeter will be installed downstream of the target to detect the photons at small angles. The target will be polarized via the method of Dynamic Nuclear Polarization (DNP) which is a well established technique that has been used extensively in nuclear and particle physics experiments.

The experiments will run for 120 days at the luminosity of $L=2 \times 10^{35} \mathrm{~cm}^{-2} \mathrm{~s}^{-1}$ using $11 \mathrm{GeV}$ electron beam provided by the upgraded accelerator of Jefferson Laboratory. The statistical uncertainties of the asymmetry measurements are expected to be between $2 \%$ and $10 \%$, depending on the value of $x_{B}$ and $Q^{2}$, because the number of expected events at low $x_{B}$ is much larger than the number of events at higher $x_{B}>0.4$. An example of the projected precision for the target-spin asymmetries assuming $80 \%$ target polarization is shown in Fig. 2. These differences between the blue dashed curve and the red solid curve clearly demonstrate the sensitivity of the target-spin asymmetry to the $\widetilde{H}$ GPD.

\section{SUMMARY}

The expected DVCS data from the experiment with a longitudinally polarized target and CLAS12 detector will extend the $Q^{2}$ range of the existing CLAS data and upcoming dedicated $6 \mathrm{GeV}$ experiment to $Q^{2}=8 \mathrm{GeV}^{2}$, at the same time providing a 

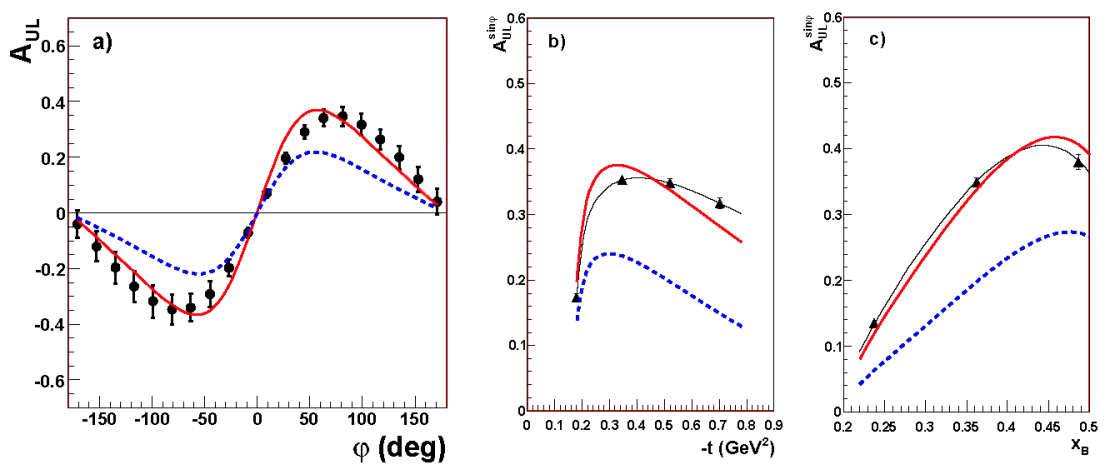

FIGURE 2. Target spin asymmetry versus $\varphi$ for $Q^{2}=4.1 \mathrm{GeV}^{2}, x_{B}=0.36$ and $-t=0.52 \mathrm{GeV}^{2}$ (a). The black points show the values from [11] using CTEQ6 PDFs with the estimated errors from the proposed measurement. The red solid curve is using MRST02 PDFs with $E=\widetilde{E}=0$, and for the blue dashed curve is $\widetilde{H}$ is also set to zero. $\sin \varphi$ moments of the target spin asymmetry versus $-t$ at $Q^{2}=4.1 \mathrm{GeV}^{2}$ and $x_{B}=0.36(\mathrm{~b})$, and versus $x_{B}$ at $Q^{2}=4.1 \mathrm{GeV}^{2}$ and $-t=$ $0.52 \mathrm{GeV}^{2}(\mathrm{c})$. The projected error bars represent the statistical uncertainties only.

large angular coverage and exceptional statistical accuracy. Along with the measurements of the beam-spin asymmetry, transversely polarized target-spin asymmetry and the helicity-dependent cross section, these data will provide a solid basis for a global fit of parametrized GPDs. With such a wide $t$ and $Q^{2}$ coverage, it will be possible to study the onset of twist-3 (and higher) effects which enter the beam-spin asymmetries and target-spin asymmetries with at least an additional power of $\sqrt{-t} / Q$.

\section{ACKNOWLEDGMENTS}

This work was supported by the U.S. Department of Energy and the National Science Foundation, the French Commissariat a l'Energie Atomique, the Italian Istituto Nazionale di Fisica Nucleare, and the Korean Science and Engineering Foundation.

\section{REFERENCES}

1. X.-D. Ji, Phys. Rev. D55, 7114-7125 (1997), hep-ph/9609381.

2. X.-D. Ji, Phys. Rev. Lett. 78, 610-613 (1997), hep-ph/9603249.

3. A. V. Radyushkin, Phys. Lett. B380, 417-425 (1996), hep-ph/9604317.

4. A. V. Radyushkin, Phys. Rev. D56, 5524-5557 (1997), hep-ph/9704207.

5. X.-D. Ji, W. Melnitchouk, and X. Song, Phys. Rev. D56, 5511-5523 (1997), hep-ph/9702379.

6. C. Munoz Camacho, et al., Phys. Rev. Lett. 97, 262002 (2006), nucl-ex/0607029.

7. F. X. Girod, et al., Phys. Rev. Lett. 100, 162002 (2008), 0711.4805.

8. A. Airapetian, et al., Phys. Rev. Lett. 87, 182001 (2001), hep-ex/0106068.

9. S. Stepanyan, et al., Phys. Rev. Lett. 87, 182002 (2001), hep-ex/0107043.

10. S. Chen, et al., Phys. Rev. Lett. 97, 072002 (2006), hep-ex/0605012.

11. M. Vanderhaeghen, P. A. M. Guichon, and M. Guidal, Phys. Rev. D60, 094017 (1999), hep-ph/ 9905372 . 